\title{
Yoga intervention and reminder e-mails for reducing cancer-related fatigue - a study protocol of a randomized controlled trial
}

\author{
Teresa Zetzl ${ }^{1 *} \mathbb{D}$, Michael Schuler ${ }^{2}$, Agnes Renner $^{1}$, Elisabeth Jentschke ${ }^{1}$ and Birgitt van Oorschot ${ }^{1}$
}

\begin{abstract}
Background: Almost $90 \%$ of cancer patients suffer from symptoms of fatigue during treatment. Supporting treatments are increasingly used to alleviate the burden of fatigue. This study examines the short-term and longterm effects of yoga on fatigue and the effect of weekly reminder e-mails on exercise frequency and fatigue symptoms.

Methods: The aim of the first part of the study will evaluate the effectiveness of yoga for cancer patients with mixed diagnoses reporting fatigue. We will randomly allocate 128 patients to an intervention group $(N=64)$ receiving yoga and a wait-list control group $(N=64)$ receiving yoga 9 weeks later. The yoga therapy will be performed in weekly sessions of 60 min each for 8 weeks. The primary outcome will be self-reported fatigue symptoms. In the second part of the study, the effectiveness of reminder e-mails with regard to the exercise frequency and self-reported fatigue symptoms will be evaluated. A randomized allocated group of the participants ("email") receives weekly reminder e-mails, the other group does not. Data will be assessed using questionnaires the beginning and after yoga therapy as well as after 6 months.
\end{abstract}

Discussion: Support of patients suffering from fatigue is an important goal in cancer patients care. If yoga therapy will reduce fatigue, this type of therapy may be introduced into routine practice. If the reminder e-mails prove to be helpful, new offers for patients may also develop from this.

Trial registration: German Clincial Trials Register (DRKS00016034, 12/2018), retrospectively registered.

Keywords: Cancer, Fatigue, Yoga, Reminder e-mails, Supportive therapy, Randomized controlled trial

\section{Background}

Cancer-related fatigue ( $\mathrm{CrF}$ ) is the most common symptom of cancer treatment. Up to $90 \%$ of oncological patients suffer from fatigue during treatment $[1,2]$. Both physiological and psychosocial factors play an important role in the development of fatigue. Often, however, no specific cause can be identified and therefore no specific treatment can be offered. In these cases, additional support services should be provided to help patients to cope with symptoms of fatigue. These range from drug treatment approaches psychosocial counseling, psychoeducation, exercise training to so-called mind-body interventions [2].

\footnotetext{
* Correspondence: Zetzl_T@ukw.de

${ }^{1}$ Interdisciplinary Center, Palliative Medicine, University Hospital Wuerzburg, Josef-Schneider-Str. 2, 97080 Wuerzburg, Germany

Full list of author information is available at the end of the article
}

$\mathrm{CrF}$ is characterized by an intense and chronic sense of tiredness and exhaustion that is not associated with previous stress and cannot completely be relieved by rest. This tiredness can be found on three levels: physical, emotional and cognitive. In order to manage the fatigue symptoms on all three levels, results of meta-analyses support a multimodal approach of cognitive, physical and emotional aspects to symptom relief [3], for example consisting of psychoeducation and mind-body intervention. Few findings on the efficacy of psychoeducative interventions covering cognitive and emotional aspects and behavioral techniques on fatigue symptoms in cancer patients exist [4-6]. With regard to mind-body interventions (physical and emotional aspects), there are predominantly findings that speak for their efficacy in fatigue. The term "mind-body intervention" refers to

(c) The Author(s). 2019 Open Access This article is distributed under the terms of the Creative Commons Attribution 4.0 International License (http://creativecommons.org/licenses/by/4.0/), which permits unrestricted use, distribution, and 
interventions that aim to strengthen self-care through active and health-promoting strategies. With regard to fatigue, the focus here is mostly on mindfulness-based procedures, meditations as well as Yoga or QiGong. A meta-analysis reports a moderate effect of mind-body interventions on fatigue symptoms [7]. Both the 'Mindfulness-Based Stress Reduction Program' (MBSR) by John Kabat-Zinn [8-10] and yoga interventions [11-14] or Pranayama [15] help reducing fatigue. Even compared to a supportive or psychoeducative group of patients with fatigue, yoga therapy showed significant effects [16]. However, there are also contradictory findings that do not support significant differences in fatigue symptoms through yoga intervention compared to a control group [17-19].

Follow-up findings for mind-body interventions dealing with fatigue vary widely. While some randomized controlled trials (RCT) show significant effects in fatigue following a mind-body intervention, but no longer after 3 months [12, 20], others found no effects immediately after an intervention, but 3 months later [21]. Lengacher et al. [22] showed significant reduction of fatigue compared to a CG after the intervention and also in a catamnesis 6 weeks later [22]. An observational study showed significant longterm effects of mind-body intervention regarding depression and general mood after 1 year, but no long term effects regarding fatigue [23].

The yoga therapy intervention we used in our study was already evaluated in previous studies. Using a randomized controlled design, Lundt et al. [24] showed that this kind of yoga therapy significantly reduce anxiety in cancer patients with mean to high effect size $(d=0.75)$. However, no effects significant effects were found for fatigue and depression [24]. In an observational, catamnestic study without control group based on the study above, 6 months after yoga intervention significant changes in fatigue $(d=0.32)$, anxiety $(d=0.33)$ and depression $(\mathrm{d}=0.40)$ were observed compared to baseline measurement [25].

An important moderator of the effect on fatigue is frequency in exercising of the patients during and after the intervention $[19,21]$. To improve exercise frequencies, participants often receive exercise $\mathrm{CDs}$ and/or exercise books at the end of yoga intervention $[11,16$, 18-20]. Documentation of their daily exercise duration in addition to the exercise $\mathrm{CD}$ has also shown to be helpful for the sustainability of the effects [21]. In the area of oncological complementary therapy, supporting participants via phone calls at weekends within an ongoing mind-body intervention helps to maintain the continuity of the exercises [26]. In the medical context, daily reminder e-mails increased adherence to medication $[27,28]$. However, there are no comparable studies on mind-body procedures to improve fatigue symptoms that use weekly reminder e-mails to promote sustainable effects.

In this study, the following research questions will be examined:

Primary research question:

1. Does an 8-week yoga intervention (IG) reduce selfreported fatigue symptoms directly after the intervention compared to a waiting-list control group (CG)? We expect an effect of $d=0.50$ in favor of the IG.

\section{Secondary research questions}

2. Do gender, age, cancer, baseline fatigue, depression and period since first diagnosis moderate the effects of the yoga intervention on self-reported fatigue?

3. Do reminder-e-mails after the yoga intervention reduce self-reported fatigue symptoms and improve exercise frequency compared to participants of the yoga-intervention receiving no reminder e-mails?

4. Is exercise frequency associated with lower selfreported fatigue symptoms?

\section{Methods}

The study will take place in the premises of the University Hospital Wuerzburg Radiotherapy/Palliative Care Unit. Written informed consent will be obtained from all participants. To answer the research questions, a combination of two RCTs will be implemented (see Fig. 1). In the first part, eligible patients will be randomized to the IG (8-week yoga intervention) or CG. The CG will also receive the yoga intervention, but nine weeks later than the IG. At the end of the yoga intervention, the second RCT will be implemented: all patients will be again randomized to get reminder-emails (email group) or no reminder emails after the yoga-interventions. The email group will receive a weekly email over 24 weeks a week, in which one asana out of the yoga intervention is repeated and described in detail. In addition, through the reminder-email participants will be encouraged to practice by themselves regularly. The first RCT is described in 2.1 and will be used to answer research questions 1 and 2, the second RCT is described in 2.2 and will be used to answer research questions 3 and 4 .

\section{First RCT (yoga intervention) Study-setting and eligibility criteria}

The participants will be at least 18 years old, will have a previous oncological disease and will undergo treatment at the radiotherapy outpatient clinic or the interdisciplinary oncological therapy outpatient clinic (IOT) at the time of screening and they report fatigue in Fischer screening (intensity $\geq 4$, impairment $\geq 5$ ). Exclusion criteria will be insufficient knowledge of German and 


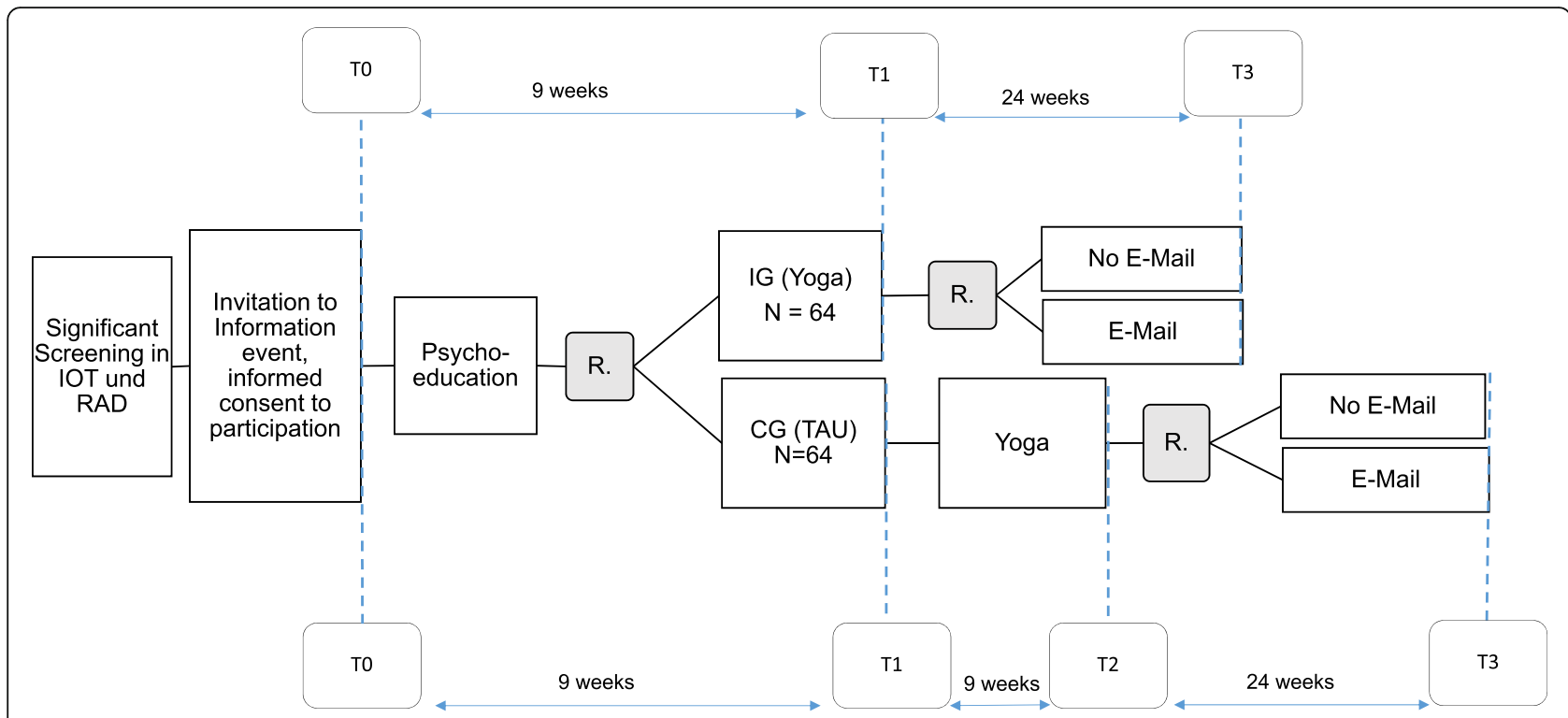

Fig. 1 Study design; Abbreviations: IOT = interdisciplinary oncological therapy outpatient clinic; RAD = radiation therapy ambulance; R= Randomization; $\mathrm{IG}=$ Intervention group, $\mathrm{CG}=$ Control group TAU = treatment as usual

severe emotional or physical impairment as well as more than $50 \mathrm{~km}$ distance to the university hospital which would require travelling.

\section{Study design and measurement occasions}

Eligible patients will receive the first set of questionnaires as a baseline assessment (T0). All participants will receive psychoeducation intervention (coping with fatigue). Subsequently, they will be randomized to IG or CG. The IG starts with the yoga intervention 1 week after T0, the CG starts 10 weeks after T0. The yoga intervention will be conducted for 8 weeks plus an additional lesson in the ninth week to give the participants the opportunity to catch up on a missed session. Primary and secondary outcomes will be assessed 10 weeks after the start of yoga intervention (T1) via questionnaires. Participants of the CG will also answer a questionnaire at the begin of the yoga intervention (T1) and at the end of the yoga intervention (T2, this will be used in the second RCT).

\section{Yoga-intervention}

Certified yoga teachers will carry out yoga intervention. The sequence of exercises will remain constant from beginning to end. The asanas (physical exercises from Yoga) were inspired by John-Kabat Zinn and adopted for cancer patients by Dr. Jentschke (psychooncologist, physiotherapist and yoga teacher). One yoga session will last 1 hour. It consists of physical exercises (asanas), conscious breathing (Pranayama) and deep relaxation (Savasana). Nonviolence (ahimsa) as an important basic principle of yoga is to be repeated every hour and helps to encourage the participants to deal gently with their bodies. Participants should not perform any physical exercises that cause pain. The subsequent body exercises are structured from lying to sitting to standing. The following sequence of exercises will be repeated in each yoga unit: 1) Relaxation: conscious breathing, body scan, mindfulness 2) Vein pump 3) Pelvis and back rotation (adapted variation of the "nakrasana") 4) Pelvis opening (adapted variation of the "supta baddha konasana") 5) Shoulder bridge ("setu bandha sarvangasana") 6) Forward folds (Paschimottanasana and variations with Pranayama) 7) Backbend: intense east stretch (Purvottasana) 8) Diagonal static yoga cat (Majariasana 1 and resting pose) 9) Standing exercise 10) Upward Salute(Urdhva Hastasana) 11) Warrior 1 (Virabhadrasana 1) 12) Warrior 3 (Virabhadrasana 3) 13) Tree (Vrkasana variation) 14) Relaxation (Savasana). In all exercises, participants are reminded to breathe slowly, deeply and consciously. Adverse events and complications during yoga therapry are recorded by yoga teachers on a standardized basis.

\section{Sample size calculation}

Two randomized intervention studies investigating yoga therapies in cancer patients of comparable type and duration using an intervention condition and a waiting-list control group condition produced intergroup effect size in fatigue self-assessment scales of $d=0.66$ [29] and $d=0.51$ [18]. Based on the lower effect size of $d=0.50$ alpha $=0.05$ and Power $=0.80$ result in a case number of $n=64$ per group, i.e. 128 patients in total, for a t-test for independent samples with two-sided testing. 


\section{Recruitment}

Oncological patients from the radiotherapy outpatient clinic and the interdisciplinary oncological therapy outpatient clinic who score high in fatigue screening will be invited to a non-binding event via mail where they will receive further information on the study. If no response is received within a week of the invitations being sent, patients will be contacted again by telephone to clarify any ambiguities. Through this recruitment, we hope to maximize participation rate in the first information event. At the first event, the patients will be explained the purpose and procedure of the study and asked to participate in the study.

\section{Randomization}

The information events will take place every 4 weeks. The participants of the study will be recruited on each information event. All patients who met inclusion criteria will be asked to participate. Participants who will have signed the consent form and completed the first questionnaire set will be randomly assigned to the IG or CG. To ensure that enough patients will be randomized to the intervention group to perform the intervention, a block randomization procedure will be used. Patients meeting the same information event will form a block. The randomization list with computer-generated numbers will be created by the Institute of Clinical Epidemiology and Biometry of the university of Wuerzburg.

\section{Second RCT (reminder e-mails)}

\section{Study design and measurement occasions}

After the yoga intervention, all participants are randomly assigned to group "Email" who will get weekly reminder e-mail or to group "NoEmail" who will receive no reminder e-mails. Participants of both groups will receive a practice book and a practice $\mathrm{CD}$. Outcomes will be assessed 6 months after the end of the yoga therapy (T3).

\section{Reminder e-mail}

Reminder e-mails are used for the second question. The e-mails in the first 12 weeks contain descriptions of the twelve asanas - one asana each week - and an encouragement to practice yoga during this week. The asanas are described analogously to the order of the yoga classes. In the following 12 weeks, the 12 reminder e-mails will be repeated in the same order.

\section{Randomization}

All participants of the Yoga study will be randomly assigned to the group "Email" or "NoEmail". A block randomization procedure will be used. Patients assigned to a group (IG or CG) after the information event form a block. The randomization list with computer- generated numbers is compiled by staff members of the interdisciplinary Palliative Medicine Centre.

\section{Outcomes, ethics, data management and statistics Outcomes and other measures}

Outcomes will be assessed using patient questionnaires or will be extracted from the patient-documentation system. Unless otherwise specified, all outcomes are recorded at all measurement times (T0, T1, T2, T3).

\section{Primary outcome: self-reported fatigue}

Self-reported fatigue will be assessed using the German version of EORTC QLQ-FA13 13 Items (European Organization for Research and Treatment of Cancer Quality of Life Questionnaire - Fatigue) [30]. This questionnaire can be used in all tumor diseases in all stages and phases of the disease and in all areas of treatment (chemotherapy, radiation, surgery) or care (acute care, rehabilitation, aftercare or palliative care) [31]. Fatigue is measured using 13 items. Response categories of all items are 'not at all', 'a little', 'quite a bit', and 'very much', coded with scores from 1 to 4 . All Items are summed up to one fatigue score. The scores range from 0 to 100 . Higher values indicate a higher level of fatigue symptoms. The internal consistency for the German version was good with Cronbach's alpha values ranging from .79-.90 [30, 32].

\section{Secondary outcomes}

Depression The Patient Health Questionnaire (PHQ-9) will be used to assess depression. The 9 items assess depression symptoms according to DSM-IV/DSM-V and are scored on a 4-point Likert scale $(0=$ not at all, $1=$ several days; $2=$ more than half of the days; $3=$ nearly all days). All items are summed up to one sum score, ranging from (0-27). Higher values indicate higher depression. The internal consistency for the PHQ-9 proved to be good with Cronbach's $\alpha=0.79$ for cancer patients [33].

Quality of life The EORTC QLQ-C15-PAL is a short form [34] of the QLQ-C30 for palliative care settings. The 15 items of the questionnaire assess nine categories: physical function, emotional function, global QoL, pain, fatigue, appetite, dyspnea, constipation, and sleep. The four response categories are coded with scores from 1 to 4 (1: not at all; 2: a little; 3 : quite a bit; 4: very much) and are transformed to a $0-100$ scale range.As an exception, global QoL is scored from 1 (very poor) to 7 (excellent). A higher score represents better function and QOL, while for symptom scale, it indicates greater symptom burden. 
Symptom assessment The German version of the Edmonton Symptom Assessment Scale (ESAS) [35] will be used to assess symptom severity. The nine symptoms pain, fatigue, nausea, sadness, anxiety, drowsiness, appetite, general condition and shortness of breath are assessed on a numerical rating scale from $0=$ none to $10=$ worst possible. Higher values in the summed scale score indicate high symptom burden. The internal consistency according to Cronbach's alpha range between $0.67-0.73$ [35].

Distress thermometer The distress thermometer is used to measure psychosocial stress. It uses a numeric rating scale ranging from 0 (no distress) to 10 (extreme distress) and is widely used in clinical practice [36]. A cut-off value of $\geq 5$ is referred to as high distress. Both the dichotomous classification and the sum score of the district thermometer are to be recorded in the study. As a short screening instrument, the Distress Thermometer is well suitable for identifying high loads recorded by Hospital Anxiety and Depression Scale $>11$ (AUC values 0.71-0.76) [36].

Sense of coherence The German version of the short form of Sense of Coherence Scale (SOC L9) [37] will be used to record the sense of coherence. This is a reliable, valid and economic alternative short form of the SOC developed by Antonovsky (1983). The 9 items are scored on an 8-point Likert scale. Response formats vary between items. Higher values indicate a high degree of coherence, whereby age- and genderdependent norms must be applied. The internal consistency of the SOC L9 can be rated as good (Cronbach's alpha $=.87$ ) [37].

Sociodemographic and health data The following socio-demographic data will be assessed at T0: age, gender, marital status, number of children, level of education, professional status. Individual coping with the tumor disease, treatment status and use of analgesics and psychopharmaceuticals will be assessed at each measurement occasion. In addition, previous experience with yoga and expectations of the participants on yoga will be asked at T0.

Program evaluation and training time At the end of the intervention (IG: T1; CG: T2), participants comprehensibility and applicability of the yoga theory and psychoeducation as well as subjective benefit will be assessed on a scale from 0 (not at all) to 3 (very). Furthermore, the current scope of the exercise duration (yoga) and the application frequency of the recommendations for handling fatigue will be measured at T1(IG)/
T2(CG) and T3(IG and CG). Reasons for continuing or ending yoga practice are documented.

\section{Data management and confidentiality}

In order to guarantee the confidentiality of data, the data will be pseudonymized using an allocation list. A research code will be assigned to each study participant so that only the code and no personal data will be used on all questionnaires. All questionnaires will be kept in locked cabinets and/or password protected computers. The allocation list will be only accessible to the data manager of the University Hospital Wuerzburg responsible for allocating medical data. The allocation list is deleted after the end of the study. It will contain the name, address, date of birth, SAP research number and contact data of the patients. During the period of this assignment, the research data will be considered "personal data" and the data protection laws are to be complied. The questionnaires will be scanned by the software EvaSys. The data will be exported to statistical programs for further statistical analysis. The data manager will compare the transferred data of the questionnaires item by item with the original questionnaires. Additional data, such as treatment duration and intensity, will be taken from the electronic patient file. The data manager will also advise on storage, back up and archiving of data to ensure databases are regularly backed up to ensure data is safeguarded from accidental loss. The study master file and all study documentation will be archived for at least 10 years.

\section{Statistical analysis}

To answer the first research question, analyses of covariance (ANCOVA) will be performed, using fatigue score T1 (primary outcome) as dependent variable, type of treatment (IG vs. CG) as independent variable and fatigue at baseline as covariate. The second research question will be analyzed by including the moderator (e.g. gender, age) and the interaction terms (for example group X gender) in the model.

The third research question will be tested again by using ANCOVA, with fatigue T3 as dependent variable, fatigue after the intervention (IG: T1, CG: T2) as covariate and type of reminder (e-mail vs. no email) as independent variable. The fourth research question will be tested by computing a mediation model using structural equation modeling framework $[38,39]$.

\section{Ethics, consent and permission}

The investigation will conform to the principles outlined in the Declaration of Helsinki. The study protocol was approved by the Ethics Committee of the University Wuerzburg on 15/05/2018 (Nr. 59/18 sc). If interested, all potential participants will receive detailed written 
information on all relevant aspects of the study. Participants will be informed that they could withdraw from the study at any given time and without reason and that participation in the study is voluntary. Participants will be assured that any future treatment will not be affected in any way should they choose to withdraw. The patients will consent to the study after detailed information. The study is registered on German Clincial Trials Register (DRKS00016034, 12/2018). This paper contains the original study protocol. Material changes to the study protocol will be submitted to the Ethics Committee of the University of Wuerzburg for approval. These changes are documented in detail in the German Register of Clinical Trials and described transparently in study reports.

\section{Discussion}

Many cancer patients report fatigue as a very negative stressful side effect at the simultaneous time strong perceived helplessness. In this study, we will offer demandoriented psychoeducation and Yoga therapy to patients of all tumor entities who suffer from fatigue. The effect of psychoeducation with yoga is compared to psychoeducation without yoga. To our knowledge, the combination of psychoeducation and yoga therapy is an innovation that follows the multidimensional approach for overcoming fatigue. Exploratively, the results of this study (yoga plus psychoeducation) can be compared with other studies (yoga only) at the Interdisciplinary Center for Palliative Medicine. By including different tumor entities, this study can provide indications as to which patients with which tumor entities can benefit most from yoga therapy. So other hypotheses can be generated, such as the degree of fatigue that patients benefit most from the intervention, whether there are differences between the different tumor types in terms of the benefit and feasibility of the intervention, or to what extent tumor therapy has an influence on the intervention.

At the same time, it is to be examined to what degree reminder e-mails are helpful for the sustainability of the practice time for yoga exercises 6 months after yoga therapy. In addition, the relationship between exercise frequency and the extent of fatigue symptoms will be examined.

If yoga therapy proves to be supportive for oncological patients and reduces fatigue, this type of therapy should continue to be introduced into routine practice. If differences in the effectiveness of yoga therapy are found in different patient groups, it should be considered whether yoga therapy needs to be adapted to specific patient needs. Further studies should also be conducted to determine the barriers to participation in yoga therapy. The aim is to develop a supportive program for oncological patients that is as adapted as possible to their needs.

If the reminder e-mails prove to be helpful with regard to exercise frequency and fatigue symptoms, new offers for patients may also develop from this. For example, the focus is on internet-based training sessions or independent yoga exercises at home.

The findings of this study will be published in peerreviewed journals and will be presented in conferences.

\section{Abbreviations}

CG: control group; CrF: Cancer related Fatigue; EORTC QLQ C15

PAL: European Organization for Research and Treatment of Cancer - Quality of Life Questionnaire - Palliative; EORTC QLQ FA: European Organization for Research and Treatment of Cancer - Quality of Life Questionnaire - Fatigue; ESAS: Edmonton Symptom Assessment Scale; IG: Intervention group; IOT: Interdisciplinary oncological therapy outpatient clinic;

MBSR: Mindfulness-based stress reduction; PHQ-9: Patient Health questionnaire; QoL: Quality of life; RCT: Randomized controlled trial; SOC: Sense of coherence

\section{Acknowledgments}

Not applicable.

\section{Authors' contributions}

VOB TZ EJ MS developed the study design. $\mathrm{VOB}$ initiated the study and obtained funding. TZ supervises the study and data collection TZ wrote the first draft of the manuscript, which was critically reviewed by MS and AR. All authors finally read and approved the manuscript for submission. TZ and AR participate in conducting the study and in data collection. MS participates in conducting the study and developing the design and statistical analysis plan.

\section{Study status}

Recruiting has started and is ongoing.

\section{Funding}

This work is supported by the Deutsche Krebshilfe, Buschstr. 32, 53113 Bonn. Funding covers costs for personal, materials and traveling expenses. As a sponsor, Deutsche Krebshilfe plays no role in the study design, collection, analysis, interpretation or writing of the manuscript. DKH has peer-reviewed the study protocol. This publication was funded by the German Research Foundation (DFG) and the University of Wuerzburg in the funding programme Open Access Publishing

\section{Availability of data and materials} Not applicable.

\section{Ethics approval and consent to participate}

The investigation conforms to the principles outlined in the Declaration of Helsinki. The study protocol was approved by the Ethics Committee of the University Würzburg on 15/05/2018 (Nr. 59/18 sc). Eligible patients will receive written information about all relevant aspects of the study and that their participation in the study is voluntary and they have the right to refuse or to withdraw their consent at any time without reprisals. Patients providewritten informed consent. The study has been registered with the German Clinical Trials Register DRKS00016034). This paper contains the original study protocol. Any substantial modifications to the study protocol will be submitted to the Ethics Committee of the University Würzburg for approval prior to implementation. These amendments will be documented in detail in the German Clinical Trials Register and will be described transparently in trial reports.

Consent for publication

Not applicable.

Competing interests

The authors declare that they have no competing interests. 


\section{Author details}

${ }^{1}$ Interdisciplinary Center, Palliative Medicine, University Hospital Wuerzburg, Josef-Schneider-Str. 2, 97080 Wuerzburg, Germany. ${ }^{2}$ Institute of Clinical Epidemiology and Biometry, University of Wuerzburg, Josef-Schneider-Str. 2, 97070 Wuerzburg, Germany.

Received: 12 July 2019 Accepted: 3 September 2019

Published online: 18 September 2019

\section{References}

1. Körner P, Ehrmann K, Hartmannsgruber J, Metz M, Steigerwald S, Flentje M, van Oorschot B. Patient-reported symptoms during radiotherapy. Strahlenther Onkol. 2017;193:570-7.

2. Berger AM, Mooney K, Alvarez-Perez A, Breitbart WS, Carpenter KM, Cella D, et al. Cancer-related fatigue, version 2.2015. J Natl Compr Canc Ne. 2015;13: 1012-39.

3. Kangas M, Bovbjerg DH, Montgomery GH. Cancer-related fatigue: a systematic and meta-analytic review of non-pharmacological therapies for cancer patients. Psychol Bull. 2008;134:700-41.

4. Reif $K$, de Vries U, Petermann F, Görres S. A patient education program is effective in reducing cancer-related fatigue: a multi-Centre randomised twogroup waiting-list controlled intervention trial. Eur J Oncol Nurs. 2013;17: 204-13.

5. Duiits SF, Mizja F, Oldenburg Hester S, van Marc B, Neil A. Effectiveness of behavioral techniques and physical exercise on psychosocial functioning and health-related quality of life in breast cancer patients and survivors-a meta-analysis. Psycho-Oncol. 2011;20:115-26.

6. Gielissen MFM, Verhagen S, Witjes F, Bleijenberg G. Effects of cognitive behavior therapy in severely fatigued disease-free cancer patients compared with patients waiting for cognitive behavior therapy: a randomized controlled trial. J Clin Oncol. 2006;24:4882-7.

7. Buffart LM, van Uffelen JGZ, Riphagen II, Brug J, van Mechelen W, Brown WJ, Chinapaw MJM. Physical and psychosocial benefits of yoga in cancer patients and survivors, a systematic review and meta-analysis of randomized controlled trials. BMC Cancer. 2012;12:559. https://doi.org/10.1186/14712407-12-559.

8. Rahmani S, Talepasand S. The effect of group mindfulness - based stress reduction program and conscious yoga on the fatigue severity and global and specific life quality in women with breast cancer. Med J Islam Repub Iran. 2015;29:175.

9. Ledesma D, Kumano H. Mindfulness-based stress reduction and cancer: a meta-analysis. Psycho-Oncol. 2008;18:571-9.

10. Kabat-Zinn J, Massion AO, Kristeller J, Peterson LG, Fletcher KE, Pbert L, et al. Effectiveness of a meditation-based stress reduction program in the treatment of anxiety disorders. Am J Psychiat. 1992;149:936-43.

11. Carson JW, Carson KM, Porter LS, Keefe FJ, Seewaldt VL. Yoga of awareness program for menopausal symptoms in breast cancer survivors: results from a randomized trial. Support Care Cancer. 2009;17:1301-9.

12. Cramer H, Rabsilber S, Lauche R, Kummel S, Dobos G. Yoga and meditation for menopausal symptoms in breast cancer survivors-a randomized controlled trial. Cancer. 2015;121:2175-84.

13. Bower JE, Greendale G, Crosswell AD, Garet D, Sternlieb B, Ganz PA, et al. Yoga reduces inflammatory signaling in fatigued breast cancer survivors: a randomized controlled trial. Psychoneuroendocrino. 2014:43:20-9.

14. Banasik J, Williams H, Haberman M, Blank SE, Bendel R. Effect of lyengar yoga practice on fatigue and diurnal salivary cortisol concentration in breast cancer survivors. J Am Acad Nurse Prac. 2011;23:135-42.

15. Chakrabarty J, Vidyasagar M, Fernandes D, Joisa G, Varghese P, Mayya S. Effectiveness of pranayama on cancer-related fatigue in breast cancer patients undergoing radiation therapy: a randomized controlled trial. Int J Yoga. 2015;8:47-53.

16. Vadiraja HS, Rao RM, Nagarathna R, Nagendra HR, Patil S, Diwakar RB, et al. Effects of yoga in managing fatigue in breast Cancer patients: a randomized controlled trial. Indian J Palliat Care. 2017;23:247-52.

17. Culos-Reed NS, Carlson LE, Daroux L, Hately-Aldous S. A pilot study of yoga for breast cancer survivors: physical and psychological benefits. PsychoOncol. 2005;15:891-7.

18. Chandwani KD, Thornton B, Perkins GH, Arun B, Raghuram NV, Nagendra HR, et al. Yoga improves quality of life and benefit finding in women undergoing radiotherapy for breast cancer. J Soc Integr Oncol. 2010;8:43-55.
19. Moadel AB, Shah C, Wylie-Rosett J, Harris MS, Patel SR, Hall CB, Sparano JA. Randomized controlled trial of yoga among a multiethnic sample of breast cancer patients: effects on quality of life. J Clin Oncol. 2007;25:4387-95.

20. Bower JE, Crosswell AD, Stanton AL, Crespi CM, Winston D, Arevalo J, et al. Mindfulness meditation for younger breast cancer survivors: a randomized controlled trial. Cancer. 2015;121:1231-40. https://doi.org/10.1002/cncr.29194.

21. Kiecolt-Glaser JK, Bennett JM, Andridge R, Peng J, Shapiro CL, Malarkey WB, et al. Yoga's impact on inflammation, mood, and fatigue in breast Cancer survivors: a randomized controlled trial. J Clin Oncol. 2014;32:1040-9.

22. Lengacher CA, Reich RR, Paterson CL, Ramesar S, Park JY, Alinat C, et al. Examination of broad symptom improvement resulting from mindfulnessbased stress reduction in breast Cancer survivors: a randomized controlled trial. J Clin Oncol. 2016;34:2827-34.

23. Kieviet-Stijnen A, Visser A, Garssen B, Hudig W. Mindfulness-based stress reduction training for oncology patients: patients' appraisal and changes in well-being. Patient Educ Couns. 2008;72:436-42.

24. Hardoerfer $\mathrm{K}$, Jentschke E. Effect of yoga therapy on symptoms of anxiety in Cancer patients. Oncol Res Treat. 2018. https://doi.org/10.1159/000488989.

25. Lundt $A$, Jentschke E. Long-term changes of symptoms of anxiety, depression, and fatigue in Cancer patients 6 months after the end of yoga therapy. Integr Cancer Ther. 2019;18:1534735418822096.

26. Banerjee B, Vadiraj HS, Ram A, Rao R, Jayapal M, Gopinath KS, et al. Effects of an integrated yoga program in modulating psychological stress and radiation-induced genotoxic stress in breast cancer patients undergoing radiotherapy. Integr Cancer Ther. 2007;6:242-50.

27. Fox MC, Creinin MD, Murthy AS, Harwood B, Reid LM. Feasibility study of the use of a daily electronic mail reminder to improve oral contraceptive compliance. Contraception. 2003;68:365-71.

28. Haramiova Z, Stasko M, Hulin M, Tesar T, Kuzelova M, Morisky DM. The effectiveness of daily SMS reminders in pharmaceutical care of older adults on improving patients' adherence to antihypertensive medication (SPPA): study protocol for a randomized controlled trial. Trials. 2017;18:334.

29. Danhauer SC, Mihalko SL, Russell GB, Campbell CR, Felder L, Daley K, Levine EA. Restorative yoga for women with breast cancer: findings from a randomized pilot study. Psycho-Oncol. 2009;18:360-8.

30. Weis J, Tomaszewski KA, Hammerlid E, Ignacio Arraras J, Conroy T, Lanceley $A$, et al. International psychometric validation of an EORTC quality of life module measuring Cancer related fatigue (EORTC QLQ-FA12). J Natl Cancer I. 2017;109.

31. Weis J. Diagnostik und Erfassung der tumorassoziierten fatigue. Forum. 2013;28:39-42.

32. Fuhrmann K, Mehnert A, Geue K, Hinz A. Fatigue in breast cancer patients: psychometric evaluation of the fatigue questionnaire EORTC QLQ-FA13. Breast Cancer. 2015;22:608-14.

33. Hartung TJ, Friedrich $M$, Johansen $C$, Wittchen $H-U$, Faller $H$, Koch $U$, et al. The hospital anxiety and depression scale (HADS) and the 9-item patient health questionnaire (PHQ-9) as screening instruments for depression in patients with cancer. Cancer. 2017:123:4236-43.

34. Groenvold M, Petersen MA, Aaronson NK, Arraras JI, Blazeby JM, Bottomley A, et al. The development of the EORTC QLQ-C15-PAL: a shortened questionnaire for cancer patients in palliative care. Eur J Cancer. 2006;42:55-64.

35. Stiel S, Matthes ME, Bertram L, Ostgathe C, Elsner F, Radbruch L. Validation of the new version of the minimal documentation system (MIDOS) for patients in palliative care: the German version of the Edmonton symptom assessment scale (ESAS). Schmerz. 2010;24:596-604.

36. Mehnert A, Müller D, Lehmann C, Koch U. Die deutsche version des NCCN distress-thermometers. Z Psychiar Psych Ps. 2006;54:213-23.

37. Schumacher J, Brähler E. Sense of Coherence Scale -Leipziger Kurzform (SOC-L9). Online-Datenbank Assessment. Institut für Qualitätssicherung in Prävention und Rehabilitation an der Deutschen Sporthochschule Köln (IQPR). 2004.

38. Preacher KJ, Hayes AF. SPSS and SAS procedures for estimating indirect effects in simple mediation models. Behav Res Meth Ins C. 2004;36:717-31.

39. Mackinnon DP, Lockwood CM, Williams J. Confidence limits for the indirect effect: distribution of the product and resampling methods. Multivariate Behav Res. 2004:39:99.

\section{Publisher's Note}

Springer Nature remains neutral with regard to jurisdictional claims in published maps and institutional affiliations. 